\title{
(2) OPEN ACCESS \\ MicroRNA expression profile in Treg cells in the course of primary immune thrombocytopenia
}

\author{
Yuandong Zhu, Huan Zhu, Xiaobao Xie, Zhuojun Zheng @ , Yun Ling
}

Department of Hematology, The Third Affiliated Hospital, Soochow University, Changzhou, China

\section{Correspondence to} Dr Zhuojun Zheng and Prof. Yun Ling, Department of Hematology, The Third Affiliated Hospital, Soochow University, Changzhou 213003, China; zenki_zheng@163.com, a110426007@hotmail.com

$\mathrm{YZ}$ and $\mathrm{HZ}$ contributed equally.

Accepted 24 May 2019 Published Online First 3 July 2019

\begin{abstract}
Primary immune thrombocytopenia (ITP) is an autoimmune bleeding disorder which characterizes with platelet production impairment and platelet destruction increment. CD4 ${ }^{+} \mathrm{CD} 25^{+}$Foxp $3^{+}$Treg cells (Tregs) are involved in the immune pathogenesis of ITP. MicroRNAs (miRNAs) are also involved in ITP and their loss of function is shown to facilitate immune disorders. Thus, the miRNA expression profile in Tregs from ITP was analyzed in this study. We assessed the genome-wide miRNA expression profile of three newly diagnosed adult patients with ITP and three healthy controls using microarray analysis of $\mathrm{CD} 4^{+} \mathrm{CD} 25^{+} \mathrm{CD} 127^{\mathrm{dim} /-}$ Tregs that were sorted using an immune magnetic bead kit. The miRNA microarray chip was based on miRBase 18.0 and Volcano Plot filtering software used to analyze the miRNA profile in Tregs. Distinct miRNA expression was further validated by fluorescence-based real-time quantitative PCR (qPCR). We found that 502 human miRNAs were differentially expressed (244 upregulated and 258 downregulated) in patients with ITP compared with healthy donors. We identified 37 miRNAs expressed significantly, including 26 upregulated and 11 downregulated. Among the deregulated miRNAs, three downregulated miRNAs including miR-155-5p, miR-146b-5p, and miR-142-3p were selected for $q P C R$ verification. We confirmed that miR-155-5p, miR-146b-5p, and miR-142-3p were significantly decreased in Tregs from patients with ITP compared with healthy controls. Compared with the healthy controls, miRNAs expressed differentially in the Tregs of patients with ITP. The levels of expression of miR-155-5p, miR-146b-5p, and miR-142-3p were significantly decreased. Therefore, the deregulation of miRNAs may affect the function of Tregs in the course of ITP.
\end{abstract}

\section{INTRODUCTION}

Primary immune thrombocytopenia (ITP) is an autoimmune disease. A decreased platelet count could be observed in ITP because of autoantibody-mediated platelet destruction and insufficient platelet production. ${ }^{1}$ Massive bleeding is a serious complication in these patients. The pathogenesis of ITP, including the immune, genetic, and environmental factors remains unclear. One pathogenic factor is a regulatory Tregs disorder that results in antiplatelet antibodies generated by $\mathrm{B}$ cells. ${ }^{2}$ Tregs are

\section{Significance of this study}

What is already known about this subject?

- Treg cells have been involved are in the immune pathogenesis of immune thrombocytopenia (ITP). Loss of function of microRNAs is shown to facilitate immune disorders. The miRNA expression profile in Tregs remains unclear.

What are the new findings?

- miR-155-5p, miR-146b-5p, and miR142-3p were significantly decreased in Tregs from patients with ITP compared with healthy.

How might these results change the focus of research or clinical practice?

- The results may provide new implication for novel therapeutic approaches for ITP.

important immune-regulating cells that maintain immune tolerance and play an important role in a variety of autoimmune diseases. ${ }^{3}$ Tregs are a specialized subpopulation of $\mathrm{T}$ cells that act to suppress the activation of the immune system and thereby maintain homeostasis and tolerance to self-antigens. Tregs are a CD4 ${ }^{+}$ subset, characterized by CD25 $5^{\text {high }}$ expression, because immunoregulatory function is absent in $\mathrm{CD} 4{ }^{+} \mathrm{CD} 25^{\text {low/neg }}$ cells. Moreover, $\mathrm{CD} 4{ }^{+} \mathrm{C}$ $\mathrm{D} 25^{\text {thigh }} \mathrm{T}$ cells are characterized by a high expression of the forkhead/winged-helix transcription factor 3 (Foxp3) gene. Patients with ITP have a Treg immunodeficiency, including a reduction in the number of Tregs and/or weakened immunosuppressive function. ${ }^{4}$

MicroRNAs (miRNAs) are short (about 22 nts) noncoding RNAs that control gene expression at the post-transcriptional level and have been proven to play critical roles in the development, differentiation, and function of immune cells. Recently, miRNAs have been shown to play an important role in the immunosuppressive function of Tregs. ${ }^{5}$ Tregs have their own particular miRNA expression profiles. Cobb et al reported that the miRNA expression profile in Treg is distinct from those in conventional $\mathrm{CD}^{+}{ }^{+} \mathrm{T}$ cells. ${ }^{6}$ Moreover, miR-155 inhibition sensitizes $\mathrm{CD}^{+}$Th cells for Treg-mediated suppression. ${ }^{7}$ We therefore intended to explore 
Table 1 RT primer of $u 6$ and miRNA

\begin{tabular}{ll}
\hline Primer name & RT primer \\
\hline U6 & 5'CGCTTCACGAATTTGCGTGTCAT3' \\
\hline miR-155-5p & 5'GTCGTATCCAGTGCGTGTCGTGGAGTCGGCAATTGCACTGGATACGACACCCCT3' \\
miR-146b-5p & 5'GTCGTATCCAGTGCGTGTCGTGGAGTCGGCAATTGCACTGGATACGACAGCCTATG3' \\
\hline miR-142-3p & 5'GTCGTATCCAGTGCGTGTCGTGGAGTCGGCAATTGCACTGGATACGACTCCATAAA3' \\
\hline
\end{tabular}

the potential correlation of miRNA during development of Treg dysfunction in the course of ITP.

\section{MATERIALS AND METHODS}

\section{Patients and health controls}

There were 21 newly diagnosed adult patients with ITP (16 females and 5 males, mean age $34 \pm 12$ years) enrolled in this study. The diagnosis of ITP was based on recently reported criteria. $^{8}$ Of the selected patients, their platelet counts ranged from 7 to $25 \times 10^{9} / \mathrm{L}$, and we excluded tumor disease, autoimmune disease, peptic ulcer, severe infection, and pregnancy.

There were 18 healthy volunteers (14 females and 4 males, mean age $35 \pm 10$ years) included as the control group with platelet counts ranged from 125 to $260 \times 10^{9} / \mathrm{L}$. The differences between two groups regarding to sex and age was not statistically significant.

The miRNA array study was carried out in 3 patients and 3 age-matched and sex-matched healthy donors and then used fluorescence-based real-time quantitative PCR (qPCR) to measure selected miRNAs from the remaining 18 patients and 15 healthy controls. This study was approved by our hospital-based ethics committee and informed consent was obtained from the patients as legal guardians.

\section{Isolation of peripheral blood mononuclear cells}

Peripheral blood was collected into EDTA-anticoagulated vacuum tubes. Peripheral blood mononuclear cells were isolated using lymph preparation density gradient centrifugation (Reagent Factory, Shanghai, China). A microscope was used to count cells.

\section{Isolation of Tregs using immune magnetic beads}

Purification of $\mathrm{CD} 4{ }^{+} \mathrm{CD} 25^{\text {thigh }} \mathrm{CD} 127^{\mathrm{dim} /-}$ regulatory cells was achieved in a two-step procedure, following the protocols of two Miltenyi Biotec kits for isolation of Tregs. Non-CD4 $^{+}$MACSxpress depletion cocktail and CD25 MicroBeads were used for labeling. In the first step, we used immunomagnetic depletion with MACSxpress Beads to remove the MACSxpress Separator non-CD4 ${ }^{+}$cells and the majority of $\mathrm{CD} 127^{\text {hi }}$ cells after erythrocytes were aggregated and sedimented. The CD25 MicroBeads may not be influenced by the magnetic field of the MACSxpress Separator (Miltenyi Biotec, Germany) because of its small nature. In the second step, anti-CD25-coated microbeads were directly labeled to the CD ${ }^{+} \mathrm{CD} 127^{\mathrm{dim} /-} \mathrm{T}$ cells. Then, $\mathrm{CD} 4{ }^{+} \mathrm{CD} 127^{\mathrm{dim} /-} \mathrm{T}$ cells in the pre-enriched $\mathrm{CD} 4^{+} \mathrm{T}$ cell fraction were isolated according to positive selection. After eluting, the eluted cells were regarded as CD $4^{+} \mathrm{CD} 25^{\text {thigh- }}$ $\mathrm{CD} 127^{\mathrm{dim} /-}$ regulatory cells for further analysis.

\section{Assessment of Treg purity}

CD4FITC/CD25APC (BD Company, USA) and eBioscience Flow Cytometry Staining buffer (eBioscience, USA) were used in two groups (experimental and negative groups). We followed the eBioscience Treg kit procedure in which cells were suspended by vortex shock and an eBioscience Fixation and Permeabilization set added. After a depleting wash and repeated removal of supernatant, cells were reacted with Foxp3 antibody. Multiparametric FACS analysis for the detection of the various markers was performed using a FACS Calibur system (BD, USA).

\section{RNA extraction}

Total RNA was extracted from Tregs using Trizol reagent (Invitrogen, Carlsbad, California, USA) and following the protocol for an miRNeasy mini kit (Qiagen, USA). The quantity of the RNA samples was assessed using an ultraviolet spectrophotometer (NanoDrop-1000 Technologies).

\section{miRNA array analyses}

The miRCURY LNA Array (miRBase 18.0) system was performed in three patients with ITP and three healthy donors as previously described. ${ }^{9}$ The RNA samples were then labeled with a miRCURY Array Power labeling kit (Exiqon, Denmark) and hybridized on a miRCURY LNA Array (miRBase 18.0) station and scanned with an Axon GenePix 4000B microarray scanner (Applied Biosystems, USA). The significance of upregulation or downregulation of miRNAs was determined with a fold change of $>1.3$ and a padj $<0.05$ calculated by Student's t-test.

\section{TaqMan qPCR for quantification of miRNAs}

Total RNA was extracted using Trizol reagent (Invitrogen) following the manufacturer's protocol. Primers (Invitrogen, Shanghai) were designed using Primer 5.0 software (tables 1 and 2). A reverse transcriptional kit (Epicentre, USA) and a TaqMan MicroRNA Assay kit (Superarray, USA) were used for qPCR assays. The manufacturer's instructions were strictly followed.

\begin{tabular}{ll}
\hline Table 2 & PCR primer of u6 and miRNA \\
\hline Primer name & RT primer \\
\hline U6 & F:5'GCTTCGGCAGCACATATACTAAAAT3' \\
& R:5'CGCTTCACGAATTTGCGTGTCAT3' \\
miR-155-5p & GSP:5'GGGTTAATGCTAATCGTGA3' \\
& R:5'TGCGTGTCGTGGAGTC3' \\
miR-146b-5p & GSP:5'GGGGGTGAGAACTGAATT3' \\
& R:5'TGCGTGTCGTGGAGTC3' \\
miR-142-3p & GSP:5'GGGGGTGTAGTGTTTCCTA3' \\
& R:5'CAGTGCGTGTCGTGGA3' \\
\hline
\end{tabular}



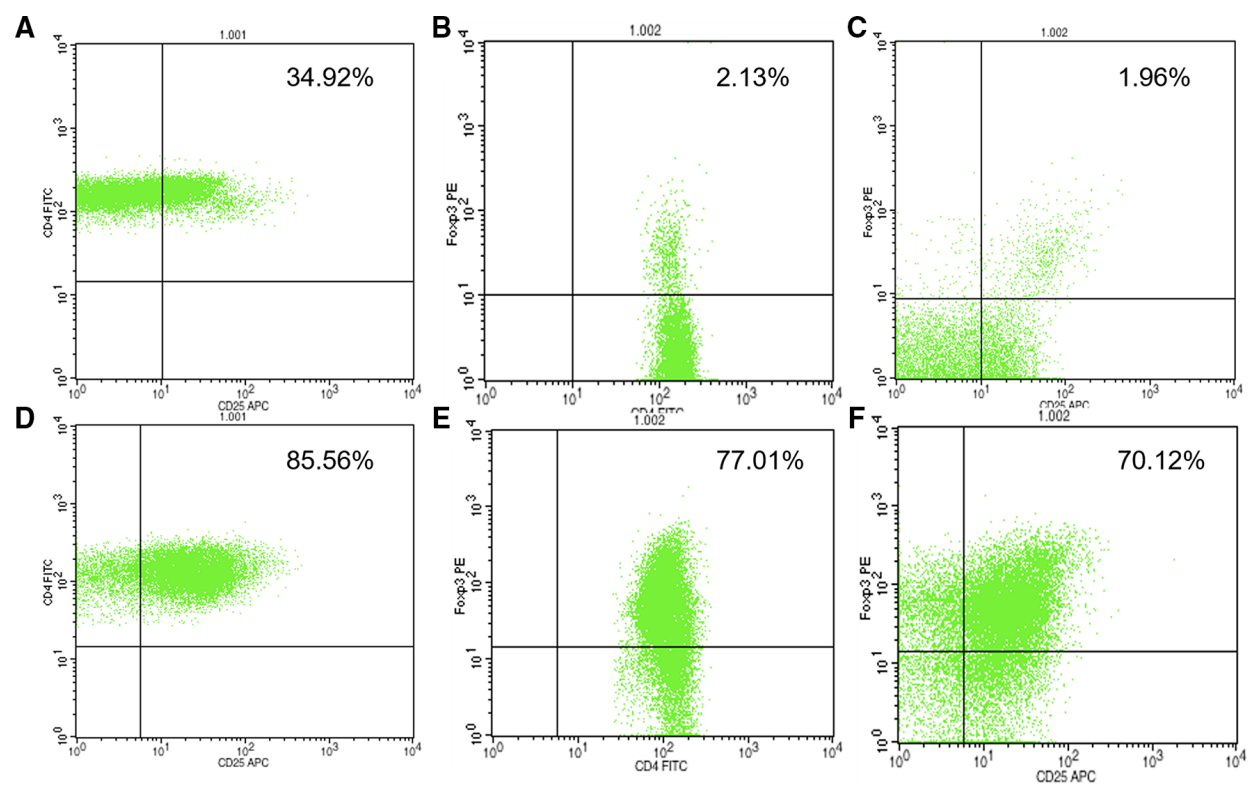

Figure $1 \mathrm{CD}^{+} \mathrm{CD} 25^{+} \mathrm{FOXP3}{ }^{+}$Treg cell analysis by FACS: $\mathrm{CD} 4^{+} \mathrm{CD} 25^{+} \mathrm{T}$ cell before sorting $(\mathrm{A})$; $\mathrm{CD}^{+} \mathrm{FOXP3^{+ }} \mathrm{T}$ cell before sorting (B); $\mathrm{CD}^{+} \mathrm{CD} 25^{+} \mathrm{FOXP3}^{+} \mathrm{T}$ cell before sorting (C); $\mathrm{CD} 4^{+} \mathrm{CD} 25^{+} \mathrm{T}$ cell after sorting (D); $\mathrm{CD} 4^{+} \mathrm{FOXP3} 3^{+} \mathrm{T}$ cell after sorting $(\mathrm{E}) ; \mathrm{CD}^{+} \mathrm{CD} 25^{+} \mathrm{FOXP3}^{+}$ T cell after sorting (F); $\mathrm{CD}^{+} \mathrm{FOXP3}^{+}$Tregs and $\mathrm{CD}^{+} \mathrm{CD}^{2} 5^{+} \mathrm{FOXP3}^{+}$Tregs were more than $80 \%$ and $70 \%$, respectively. FACS, fluorescence activated cell sorter.

U6 small nuclear RNA was quantified as a control to normalize differences in total RNA levels.

PCRs were amplified on an ABI Prism-7900 Sequence Detection System (Applied Biosystems, USA). An initial denaturation at $95^{\circ} \mathrm{C}$ for 10 min was followed by 40 cycles of denaturation at $95^{\circ} \mathrm{C}$ for $10 \mathrm{~s}$, and extension at $60^{\circ} \mathrm{C}$ for $1 \mathrm{~min}$. A dissolution curve was drawn to confirm that the PCR specific product was as specified.

\section{Statistics}

Differences in the expression of miRNAs were analyzed using Volcano Plot software, and clustering was analyzed using MEV software (V.4.6, TIGR).

The relative quantity of gene expression was obtained by comparing the relative expression of $\mathrm{U} 6$ using a $2^{-}$ $\Delta \Delta \mathrm{Ct}$ method $\left(\mathrm{Ct}_{\text {sample }}-\mathrm{Ct}_{\mathrm{ub}}\right)$. A mean $\pm \mathrm{SE}$ error or median were used to describe numerical data. The relative levels of expression of miRNAs were analyzed using a nonparametric Mann-Whitney U test and Spearman correlation analysis. IBM SPSS Statistics for Windows, V.18.0 was used for statistical processing and $p<0.05$ was considered statistically significant.

\section{Statistical analysis}

Statistical analysis was applied by using SAS V.6.12 (SAS Institute, Cary, North Carolina, USA). The miRNAs array data were analyzed by using Volcano Plot software, and the cluster was analyzed by using MEV software (V.4.6, TIGR). The $2^{-\triangle C T}$ method was used to analyze the relative changes in gene expression from real-time quantitative PCR experiments. A median and IQR was presented to describe data. Correlation analysis of miRNAs were calculated by Spearman rank correlation coefficients. $\mathrm{P}<0.05$ was considered statistically significant.

\section{RESULTS}

\section{Assessment of Treg purity}

Purified Tregs were analyzed for their surface expression of CD4, CD25, Foxp 3 antigens, and the cell counts were about $1-2 \times 10^{5}$. FACS analysis showed that $\mathrm{CD}^{+} \mathrm{Foxp}^{+}$Tregs were more than $80 \%$ and $\mathrm{CD} 4{ }^{+} \mathrm{CD} 25^{+} \mathrm{Foxp}^{+}$Tregs were more than $70 \%$ (figure 1 ).

\section{Differential expression of miRNAs in Tregs of patients with ITP compared with healthy controls}

We chose qualified RNA for microarray and found no signal in negative controls. Experiment quality and sample properties were assessed by the correlation of gene hybridization signal (figure 2). The figure shows an increased, but not strong hybridization signal. One reason for this outcome may be the quality of miRNAs; other reasons might include the weak expression of some miRNAs in the sample or the presence of as yet unknown genes.

We assessed the levels of expression of human miRNAs in Tregs from three patients with ITP and three healthy donors using an miRCURY LNA Array (figures 3 and 4). We found that 502 genes were expressed differentially; 244 had increased expression and 258 decreased expression. Because too few transcripts showed a foldchange $>1.3$, genes that reached the significance level of $\mathrm{p}<0.05$ and with a fold-change $>1.3$ were analyzed further. We identified 37 miRNAs differentially expressed in Tregs from patients with ITP compared with healthy controls (tables 3 and 4). Among them, 26 miRNAs were statistically upregulated and 11 were statistically downregulated.

Decreased expression level of miR-155-5p, miR-146b-5p, and miR-142-3p in Tregs of patients with ITP miRNA 

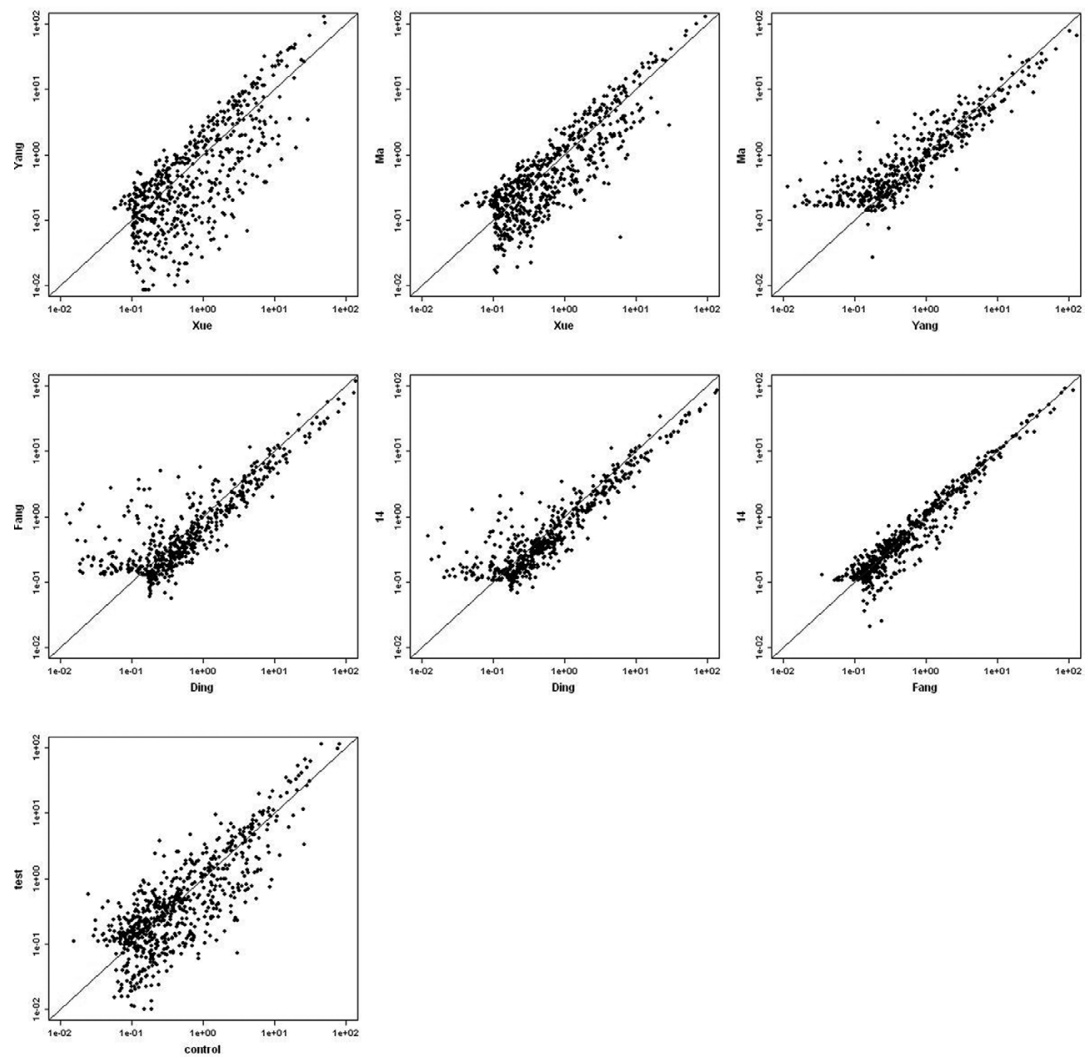

Figure 2 Correlation scatter plot of gene hybridization signal in microarray. The figure shows an increased, but not strong hybridization signal.

microarray analysis revealed that 37 aberrant miRNAs were identified. We validated the results by TaqMan qPCR in 18 patients with ITP and 15 healthy controls and found three abnormally expressed miRNAs in Tregs from patients with ITP (table 5, figure 5). Then, we compared the level of expression of miRNA in Tregs by correlation analysis and found none were significantly different (table 6).

\section{DISCUSSION}

The expression of CD25 and the Treg-specific Foxp3 were the characteristics of Treg which is essential for basic function. ${ }^{10}$ Severe inflammation and autoimmunity could be observed in mice and humans with dysfunctional Tregs, indicating that Tregs may help prevent harmful autoimmune responses. Dysregulation of Tregs could also
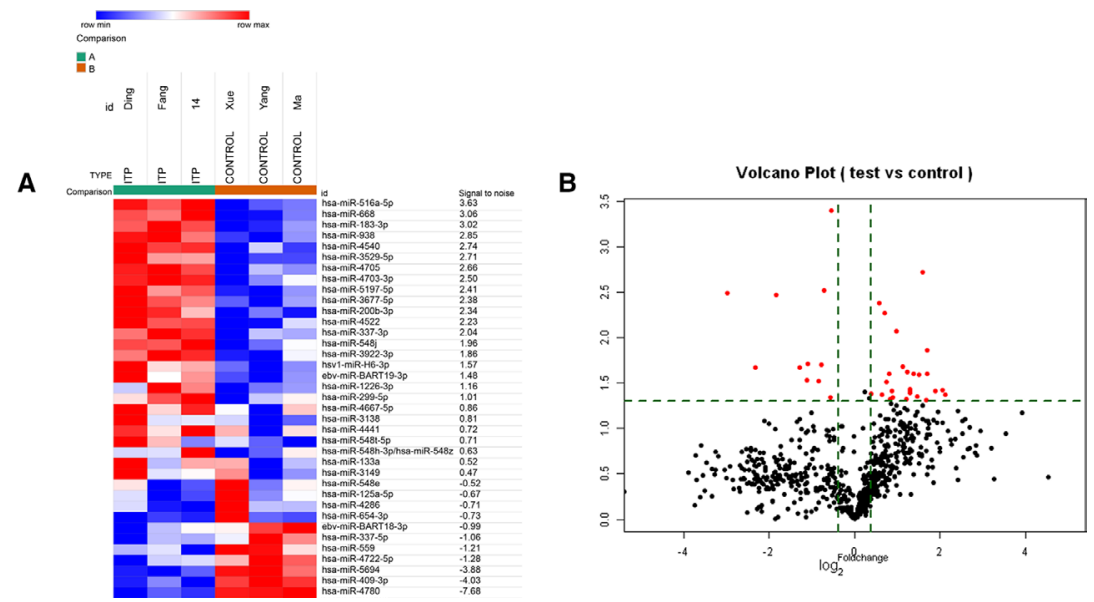

Figure 3 miRNAs clustering analysis by miRCURY LNA Array in three patients with ITP and three healthy donors. Among these, 26 miRNAs were upregulated and 11 were downregulated by a t test using a 1.3-fold change analysis. (A) The heat map of the 37 differentially expressed miRNAs between ITP samples and control samples ( $>1.3$-fold; $p<0.05)$. (B) miRNAs with fold change $>1.3$ and $\mathrm{p}<0.05$ were plotted in the volcano plot. ITP, immune thrombocytopenia. 


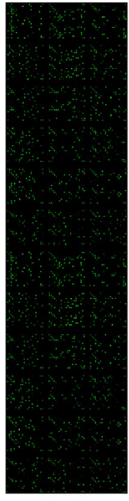

A

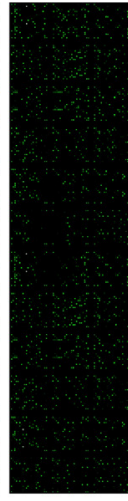

B

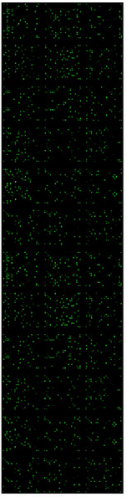

C

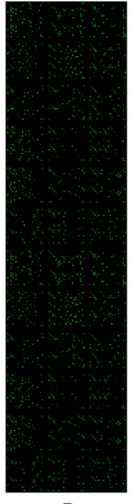

D

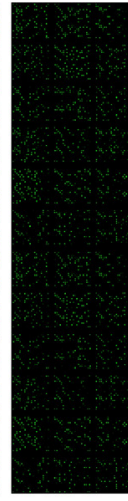

E

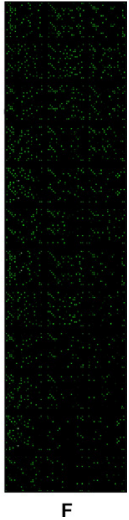

F

Figure 4 Scanning image of MicroRNA array in Tregs.

$(A-C)$ Healthy controls; $(D-F)$ patients with primary immune thrombocytopenia.

be considered to associate with ITP pathophysiology. A decreased frequency of Tregs in peripheral blood is found in patients with ITP, ${ }^{41}$ and their immunosuppressive function was inferior in patients with ITP compared with healthy controls. $^{12} 13$ In the present study, we sorted Tregs from patients with ITP and healthy controls using immunoreactive magnetic beads to study the pathogenesis of ITP and dysfunction in Tregs.

It has become more clear that miRNAs form a complex signaling pathway and aberrant expression of miRNAs is implicated in the pathogenesis of autoimmune diseases. ${ }^{14} 15$

Table 3 Upregulated miRNAs by miRNAs array

\begin{tabular}{lll}
\hline miRNA & Fold change & P value \\
\hline hsv1-miR-H6-3p & 4.134943 & 0.037759 \\
\hline hsa-miR-200b-3p & 3.214671 & 0.025018 \\
\hline hsa-miR-668 & 2.985206 & 0.001874 \\
\hline hsa-miR-548j & 2.359644 & 0.023893 \\
\hline hsa-miR-3138 & 2.743772 & 0.044483 \\
\hline ebv-miR-BART19-3p & 3.184072 & 0.04792 \\
\hline hsa-miR-3677-5p & 2.444652 & 0.036854 \\
\hline hsa-miR-3529-5p & 4.319884 & 0.042622 \\
\hline hsa-miR-133a & 1.49291 & 0.004163 \\
\hline hsa-miR-5197-5p & 2.819095 & 0.025433 \\
\hline hsa-miR-4540 & 2.454396 & 0.039986 \\
\hline hsa-miR-4667-5p & 1.752593 & 0.02491 \\
\hline hsa-miR-548h-3p/hsa-miR-548z & 1.660791 & 0.030347 \\
\hline hsa-miR-183-3p & 2.171599 & 0.020913 \\
\hline hsa-miR-4522 & 2.317606 & 0.047492 \\
\hline hsa-miR-548t-5p & 1.306963 & 0.040787 \\
\hline hsa-miR-3922-3p & 1.762495 & 0.047063 \\
\hline hsa-miR-4441 & 1.823415 & 0.038232 \\
\hline hsa-miR-337-3p & 1.962377 & 0.008488 \\
\hline hsa-miR-516a-5p & 3.215864 & 0.013648 \\
\hline hsa-miR-4703-3p & 2.448984 & 0.038187 \\
\hline hsa-miR-4705 & 2.582836 & 0.025136 \\
\hline hsa-miR-938 & 3.702528 & 0.038265 \\
\hline hsa-miR-299-5p & 1.634267 & 0.005265 \\
\hline hsa-miR-1226-3p & 1.859578 & 0.045089 \\
\hline hsa-miR-3149 & 1.557769 & 0.04242 \\
\hline
\end{tabular}

\begin{tabular}{|c|c|c|}
\hline miRNA & Fold-change & $P$ value \\
\hline hsa-miR-409-3p & 0.281322 & 0.003337 \\
\hline hsa-miR-125a-5p & 0.6731 & 0.044941 \\
\hline hsa-miR-548e & 0.560181 & 0.029698 \\
\hline hsa-miR-5694 & 0.408008 & 0.021335 \\
\hline hsa-miR-559 & 0.611151 & 0.003017 \\
\hline hsa-miR-654-3p & 0.198366 & 0.021035 \\
\hline hsa-miR-4780 & 0.126252 & 0.003205 \\
\hline hsa-miR-4286 & 0.46207 & 0.029317 \\
\hline hsa-miR-337-5p & 0.470205 & 0.0192 \\
\hline ebv-miR-BART18-3p & 0.686011 & 0.000396 \\
\hline hsa-miR-4722-5p & 0.585287 & 0.019703 \\
\hline
\end{tabular}

miRNAs also play a part in growth development, maintaining immune function and inhibiting the inflammatory response in Tregs. ${ }^{16-18}$ At present, the association of miRNAs with ITP is poorly understood ${ }^{19-21}$ and there is no report regarding miRNA microarray analysis of Tregs from patients with ITP. Among results of miRNAs tested, we found by clustering analysis that 37 were differentially expressed in Tregs from patients with ITP compared with healthy controls. We concluded that miRNAs contribute to the pathogenesis of ITP by disrupting the function of Tregs. However, the immune regulation by miRNAs is complicated and elucidating the specific mechanism requires further study.

Our previous studies confirmed that Treg cells were involved in the pathogenesis of ITP. We found that the number of Treg cells in peripheral blood of patients with ITP was significantly lower than that of normal control group, and the immunosuppressive function of Treg cells was significantly weakened. In this experiment, although 37 kinds of miRNAs were abnormally expressed, combined with previous literature, it was reported that miR-155-5p, miR-146b-5p, and miR-142-3p may be involved in regulating the development and function of Treg cells. On the other hand, as the limited sample size of patients, we initially selected miR-155-5p, miR-146b-5p, and miR-142-3p in patients with ITP for qPCR verification. We confirmed by qPCR that miR-155-5p, miR-146b-5p, and miR-142-3p were downregulated in Tregs of patients with ITP compared with healthy controls. miR-155 is located in exon 3 of chromosome 21 and is regulated by cytokines expressed by $\mathrm{T}$ cells, including IL- $10^{22}$ and TGF- $\beta .^{23} \mathrm{~A}$ suppressor of cytokine signaling (SOCS1) and signal transduction activated factor are targets of miR-155. ${ }^{162425}$ Kohlhaas $^{26}$ reported that miR-155-deficient mice have reduced numbers of Tregs, both in the thymus and periphery, because of impaired development and increasing miR-155

Table 5 Relative expression level of miRNAs

\begin{tabular}{llrl}
\hline \multirow{2}{*}{ miRNA } & \multicolumn{2}{l}{ Relative expression level } & \\
\cline { 2 - 3 } & Healthy control & Patient with ITP & P value \\
\hline miR-155-5p & $6.633(4.03-9.53)$ & $3.390(1.68-5.44)$ & 0.003 \\
miR-146b-5p & $0.08(0.07-0.11)$ & $0.015(0.01-0.05)$ & 0.001 \\
miR-142-3p & $0.36(0.10-0.42)$ & $0.08(0.05-0.22)$ & 0.002 \\
\hline
\end{tabular}



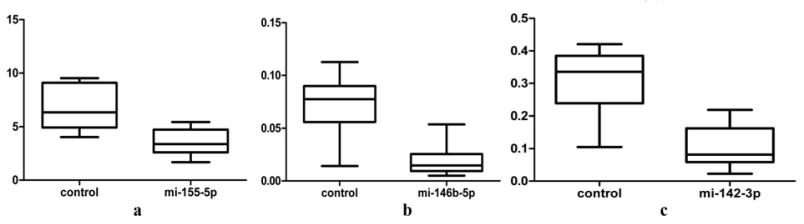

Figure 5 miRNA array results were verified by $q P C R$ inTregs of 18 patients with primary ITP and 15 healthy controls. All miRNAs were abnormally expressed in Tregs of patients with ITP. ITP, immune thrombocytopenia.

level that contributes to enhancing the immunosuppressive function of Tregs. These findings indicate that miR-155 contributes to the function and development of Tregs. We found that miR-155 was significantly downregulated in Tregs, which may lead to Treg dysfunction and contribute to ITP pathogenesis. miR-146 is composed of miR-146a and miR-146b, which locate on chromosomes 5 and 10, and have only two bases different between them. Therefore, it is not surprising that their mechanism of action and target gene are similar. ${ }^{27}$ miR-146 inhibits tumor necrosis factor receptor-related factor 6 and interleukin-1 receptorassociated kinase 1 at a post-transcriptional level through $\mathrm{NF}-\kappa \mathrm{B}$ signaling pathways and negatively regulates immune signal transduction. ${ }^{28}$ miR-146a contributes to the development of Tregs and to maintaining their immunosuppression function. We found that miR-146a is significantly decreased in peripheral blood cells from patients with ITP. We know that in patients with ITP, T helper Type 1 (Th1) cells are in a polarization mode, and damaging Treg function results in deficiencies of IFN $\gamma$, IL-10, and other cytokines. Lu et a ${ }^{29}$ reported that miR-146a, an miRNA prevalently expressed in Tregs, is critical for their suppressor function. A breakdown of immunological tolerance was led by a deficiency of miR-146a in Treg cells which manifested in fatal IFN $\gamma$-dependent immune-mediated lesions in a variety of organs. Augmented expression and activation of signal transduction and activator transcription 1 (Stat1), a direct target of miR-146a may be the crime culprit. Similarly, heightened Stat 1 activation in Tregs subjected to a selective ablation of SOCS1, a key negative regulator of Stat1 phosphorylation downstream of the IFN- $\gamma$ receptor, was associated with analogous Th1-mediated pathology-associated autoimmunity. miR-146b is also expressed abnormally in many tumor diseases. $^{30-32}$ The literature regarding miR-146 and ITP is scant. In the present microarray and PCR experiments, we found that miR-146b was significantly decreased in Tregs from patients with ITP compared with healthy controls and so we postulated that miR-146 is involved in the immunoregulation mechanism of Tregs in ITP. miR-142-3p is located in exon 1 on chromosome 17 and miR-142-3p-dependent

\begin{tabular}{|c|c|c|c|c|c|c|}
\hline \multirow[b]{2}{*}{ miRNA } & \multicolumn{2}{|c|}{ miR-155-5p } & \multicolumn{2}{|c|}{ miR-146b-5p } & \multicolumn{2}{|c|}{ miR-142-3p } \\
\hline & $r$ & $P$ value & $r$ & $P$ value & $r$ & $P$ value \\
\hline miR-155-5p & - & & & & 0.026 & 0.92 \\
\hline $\operatorname{miR}-146 b-5 p$ & 0.013 & 0.96 & & & & \\
\hline miR-142-3p & - & & 0.432 & 0.073 & & \\
\hline
\end{tabular}

functions have been identified in hematopoiesis, ${ }^{33}$ the immune system, and in relation to hemato-oncological diseases. ${ }^{34}$ miR-142-3p also has a certain relationship with Tregs. Huang et $a l^{35}$ found that Tregs exert their suppressor function by transferring cyclic adenosine monophosphate (cAMP) to responder $\mathrm{T}$ cells. miR-142-3p regulates the production of cAMP by targeting adenylyl cyclase (AC) 9 messenger RNA in Tregs. miR-142-3p limits the level of cAMP in Tregs by inhibiting AC9 production, whereas Foxp3 downregulates miR-142-3p to keep the AC9/cAMP pathway active in Tregs. We can therefore conclude that miR-142-3p regulates Tregs activity in the course of ITP. miR-142-3p has been reported in autoimmune diseases such as systemic lupus erythematous, but has not been reported to be associated with ITP. Using microarray and qPCR, we confirmed that miR-142-3p is deregulated in ITP.

In summary, we found decreased miR-155-5p, miR146b-5p, and miR-142-3p in Tregs from patients with ITP. Our data indicate that the abnormal expression of miRNAs in Tregs might play a role in the pathogenesis of ITP. However, an individual miRNA may target multiple genes. Therefore, the function of miRNA in ITP could be far more complex. The mechanisms of aberrant miRNA expression on specific immune cell subsets in ITP warrant further study.

Contributors All authors collaborated in the collection and interpretation of the data and contributed to the manuscript. YZ and $\mathrm{HZ}$ wrote the manuscript and prepared figures. XX, ZZ and YL edited the manuscript.

Funding This work was supported by grants from Changzhou Science and Technology Project (Applied Based Research, No. CJ20179025).

Competing interests None declared.

Patient consent for publication Not required.

Provenance and peer review Not commissioned; externally peer reviewed. Data sharing statement Data are available on reasonable request.

Open access This is an open access article distributed in accordance with the Creative Commons Attribution 4.0 Unported (CC BY 4.0) license, which permits others to copy, redistribute, remix, transform and build upon this work for any purpose, provided the original work is properly cited, a link to the licence is given, and indication of whether changes were made. See: https:// creativecommons.org/licenses/by/4.0/.

\section{ORCID iD}

Zhuojun Zheng http://orcid.org/0000-0003-3277-9998

\section{REFERENCES}

1 Wang T, Wang Z, Yang R. Thrombopoietic growth factors in the treatment of immune thrombocytopenic purpura. Crit Rev Oncol Hematol 2011;77:172-83.

2 Stasi R, Cooper N, Del Poeta G, et al. Analysis of regulatory T-cell changes in patients with idiopathic thrombocytopenic purpura receiving $B$ cell-depleting therapy with rituximab. Blood 2008;112:1147-50.

3 Tang Q, Bluestone JA. Regulatory T-cell physiology and application to treat autoimmunity. Immunol Rev 2006;212:217-37.

4 Yu J, Heck S, Patel V, et al. Defective circulating CD25 regulatory T cells in patients with chronic immune thrombocytopenic purpura. Blood 2008;112:1325-8.

5 Tang $X$, Tang $R$, Xu Y, et al. MicroRNA networks in regulatory T cells. J Physiol Biochem 2014;70:869-75.

6 Cobb BS, Hertweck A, Smith J, et al. A role for Dicer in immune regulation. J Exp Med 2006;203:2519-27.

7 Stahl HF, Fauti T, Ullrich N, et al. miR-155 inhibition sensitizes CD4+ Th cells for TREG mediated suppression. PLoS One 2009;4:e7158.

8 Rodeghiero F, Stasi R, Gernsheimer T, et al. Standardization of terminology, definitions and outcome criteria in immune thrombocytopenic purpura of adults and children: report from an international working group. Blood 2009;113:2386-93. 
9 Li S, Zhu J, Zhang W, et al. Signature microRNA expression profile of essential hypertension and its novel link to human cytomegalovirus infection. Circulation 2011;124:175-84.

10 Sakaguchi S, Yamaguchi T, Nomura T, et al. Regulatory T cells and immune tolerance. Cell 2008;133:775-87.

11 Aboul-Fotoh L-M, Abdel Raheem MM, El-Deen MA, et al. Role of CD4+CD25+ T cells in children with idiopathic thrombocytopenic purpura. J Pediatr Hematol Oncol 2011;33:81-5.

12 Liu B, Zhao H, Poon MC, et al. Abnormality of CD4(+)CD25(+) regulatory T cells in idiopathic thrombocytopenic purpura. Eur J Haemato/ 2007;78:139-43.

13 Bao W, Bussel JB, Heck S, et al. Improved regulatory T-cell activity in patients with chronic immune thrombocytopenia treated with thrombopoietic agents Blood 2010;116:4639-45.

14 De Santis G, Ferracin M, Biondani A, et al. Altered miRNA expression in T regulatory cells in course of multiple sclerosis. J Neuroimmunol 2010;226(12):165-71.

15 Cui Y. In silico mapping of polymorphic miRNA-mRNA interactions in autoimmune thyroid diseases. Autoimmunity 2014;47:327-33.

16 Yao R, Ma YL, Liang W, et al. MicroRNA-155 modulates Treg and Th17 cells differentiation and Th17 cell function by targeting SOCS1. PLoS One 2012; 7:e46082.

17 Singh Y, Garden OA, Lang F, et al. MicroRNA-15b/16 Enhances the Induction of Regulatory T Cells by Regulating the Expression of Rictor and mTOR. J Immunol 2015; 195:5667-77.

18 Li S, Fan Q, He S, et al. MicroRNA-21 negatively regulates Treg cells through a TGF- $\beta 1 /$ Smad-independent pathway in patients with coronary heart disease. Cell Physiol Biochem 2015;37:866-78.

19 Borjigin M, Hou W, Gong C, et al. Profiling of miRNA expression in immune thrombocytopenia patients before and after Qishunbaolier (QSBLE) treatment. Biomed Pharmacother 2015;75:196-204.

20 Bay A, Coskun E, Oztuzcu S, et al. Plasma microRNA profiling of pediatric patients with immune thrombocytopenic purpura. Blood Coagul Fibrinolysis 2014;25:379-83.

$21 \mathrm{Li} \mathrm{H}$, Zhao H, Xue F, et al. Reduced expression of MIR409-3p in primary immune thrombocytopenia. Br J Haematol 2013;161:128-35.

22 Cheung ST, So EY, Chang D, et al. Interleukin-10 inhibits lipopolysaccharide induced miR-155 precursor stability and maturation. PLoS One 2013;8:e71336.
23 Das LM, Torres-Castillo MD, Gill T, et al. TGF- $\beta$ conditions intestinal T cells to express increased levels of miR-155, associated with down-regulation of IL-2 and itk mRNA. Mucosal Immunol 2013;6:167-76.

24 Lu C, Huang $X$, Zhang $X$, et al. miR-221 and miR-155 regulate human dendritic cell development, apoptosis, and IL-12 production through targeting of p27kip1, KPC1, and SOCS-1. Blood 2011;117:4293-303.

25 Kohanbash G, MicroRNAs OH. and STAT interplay. Seminars in cancer biology 2012;22:70-5.

26 Kohlhaas S, Garden OA, Scudamore C, et al. Cutting edge: the Foxp3 target miR-155 contributes to the development of regulatory T cells. J Immunol 2009;182:2578-82.

27 Stanczyk J, Pedrioli DM, Brentano F, et al. Altered expression of MicroRNA in synovial fibroblasts and synovial tissue in rheumatoid arthritis. Arthritis Rheum 2008;58:1001-9.

28 Liu R, Liu C, Chen D, et al. FOXP3 Controls an miR-146/NF-kB Negative Feedback Loop That Inhibits Apoptosis in Breast Cancer Cells. Cancer Res 2015;75:1703-13.

29 Lu LF, Boldin MP, Chaudhry A, et al. Function of miR-146a in controlling Treg cell-mediated regulation of Th1 responses. Cell 2010;142:914-29.

30 Hurst DR, Edmonds MD, Scott GK, et al. Breast cancer metastasis suppressor 1 up-regulates miR-146, which suppresses breast cancer metastasis. Cancer Res 2009:69:1279-83.

31 Riesco-Eizaguirre G, Wert-Lamas L, Perales-Patón J, et al. The miR-146b-3p/ PAX8/NIS Regulatory Circuit Modulates the Differentiation Phenotype and Function of Thyroid Cells during Carcinogenesis. Cancer Res 2015;75:4119-30.

32 Walker SR, Xiang M, Frank DA. STAT3 Activity and Function in Cancer: Modulation by STAT5 and miR-146b. Cancers 2014;6:958-68.

33 Bissels U, Wild S, Tomiuk S, et al. Combined characterization of microRNA and mRNA profiles delineates early differentiation pathways of CD133+ and CD34+ hematopoietic stem and progenitor cells. Stem Cells 2011;29:847-57.

34 Flamant S, Ritchie W, Guilhot J, et al. Micro-RNA response to imatinib mesylate in patients with chronic myeloid leukemia. Haematologica 2010;95:1325-33.

35 Huang B, Zhao J, Lei Z, et al. miR-142-3p restricts CAMP production in CD4+CD25- T cells and CD4+CD25+ TREG cells by targeting AC9 mRNA. EMBO Rep 2009;10:180-5.

36 Buckner JH. Mechanisms of impaired regulation by CD4(+)CD25(+)FOXP3(+) regulatory $T$ cells in human autoimmune diseases. Nat Rev Immunol 2010;10:849-59. 